\title{
Deflection of Slow Light by Magneto-Optically Controlled Atomic Media
}

\author{
D.L. Zhou ${ }^{1}$ - Lan $\mathrm{Zhou}^{2}$, R.Q. Wang ${ }^{1}$, S. Yi ${ }^{2}$, and C.P. Sun讯 \\ ${ }^{1}$ Institute of Physics, Chinese Academy of Sciences, Beijing 100080, China and \\ ${ }^{2}$ Institute of Theoretical Physics, Chinese Academy of Sciences, Beijing 100080, China
}

\begin{abstract}
We present a semi-classical theory for light deflection by a coherent $\Lambda$-type three-level atomic medium in an inhomogeneous magnetic field or an inhomogeneous control laser. When the atomic energy levels (or the Rabi coupling by the control laser) are position-dependent due to the Zeeman effect by the inhomogeneous magnetic field (or the inhomogeneity of the control field profile), the spatial dependence of the refraction index of the atomic medium will result in an observable deflection of slow signal light when the electromagnetically induced transparency happens to avoid medium absorption. Our theoretical approach based on Fermat's principle in geometrical optics not only provides a consistent explanation for the most recent experiment in a straightforward way, but also predicts the new effects for the slow signal light deflection by the atomic media in an inhomogeneous off-resonant control laser field.
\end{abstract}

PACS numbers: 05.30.Ch, 03.65.-w, 05.20.Gg

\section{INTRODUCTION}

Many optical phenomena in nature, such as mirage and rainbow, can be explained in terms of refraction of light rays in an inhomogeneous optical medium [1]. The theoretical approach can be developed from Fermat's principle, saying that a light ray with a given frequency traverses the path between two points which takes the least time. Fermat's principle is consistent with light traveling in a straight line in a medium with homogenous refraction index. Classically, refraction of light results from the spatial inhomogeneity of refraction index caused by the inhomogeneity of medium density. In this article we show that the quantum coherence of optical medium even with homogenous density can also result in a spatially inhomogeneous refraction index and thus various phenomena on refraction of light.

Light deflection by an atomic medium in external fields has been studied experimentally in the last two decades [2, 3, 4, 5]. A most recent experiment with a rubidium atomic gas [6] was carried out to demonstrate how the electromagnetically induced transparency (EIT) [7, 8] enhances the light deflection in a $\Lambda$ - type three-level atomic medium. Remarkably, if a magnetic field with some gradient is applied to the gas cell, and the signal light and the control light satisfies the frequency matching condition to realize the EIT, the signal light will transmit perfectly through the atomic media with a very slow group velocity, and thus be deflected with an angle proportional to its propagation time through the gas cell. A signal light can be deflected not only by atomic media in a nonuniform magnetic field, but also by an atomic medium coherently driven by an inhomogeneous laser field, which is shown in another recent experiment [9]. As an ultra-dispersive optical prism, such coherence enhanced media has an angular dispersion which is six orders of magnitude higher than that of a prism made of optical glass.

The light deflection phenomenon observed in Ref. [6] was explained in terms of the dark polariton concept [10, 11], in which the quantized signal light field dresses the atomic collective excitation to form a quasi-particle - the polariton with an effective magnetic moment. Then, the experimental result could be understood as a Stern-Gerlach experiment for the quasi-particle. It worths noticing that, in this explanation, the signal light must be assumed as a quantized field.

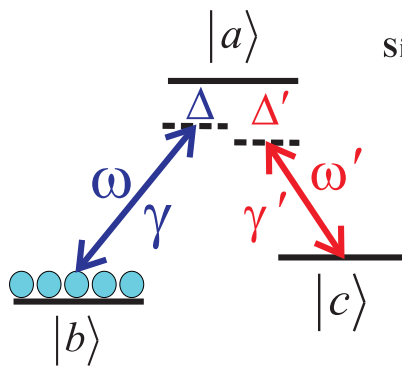

(a)

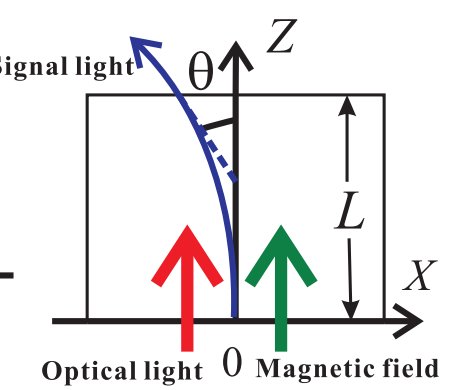

(b)
FIG. 1: (Color Online) (a) Atomic level configuration: three levels are coupled by a signal field and a control field with detunnings $\Delta$ and $\Delta^{\prime}$ respectively. (b) Schematic diagram of light deflection in the atomic medium: a transverse magnetic field gradient causes inhomogeneity of the refraction index of the medium via EIT and then deflection of the signal light beam.

In this paper we present a semi-classical theory to uniformly treat light deflection phenomena by a coherent $\Lambda$-type three-level atomic medium in various external fields. Note that the EIT enhances spatial dependence of the refraction index of the atomic medium, which results from the inhomogeneity of the magnetic field or the control laser field. We thus apply the Fermat's principle to calculate the signal light path in such coherent medium where the quantization of the signal light is not necessary to account for the experiments.

Our theory is semi-classical since the atoms are described quantum mechanically, while both the two laser fields are treated classically. This approach not only provides a consistent explanation for the experiment [6] in a straightforward way, but also predicts the new effects for the weak light deflection by an atomic media driven by an optical laser with inhomogeneous profile. This situation with weak probe light is 
essentially different from the current experiment about "ultradispersive optical prism" [9], where the strength of the probe light is as strong as that of the control light, and thus the linear suspensibility obtained for weak probe light will not be enough to account for the data of the experiment.

\section{SEMI-CLASSICAL APPROACH FOR LIGHT DEFLECTION}

As shown in Fig. 1 (a), the system under consideration is an atomic gas cell filled with $\Lambda$-type three-level atoms with an upper level $|a\rangle$ and two lower levels $|b\rangle$ and $|c\rangle$. The radiative decay rates from $|a\rangle$ to $|b\rangle$ and from $|a\rangle$ to $|c\rangle$ are, respectively, $\gamma$ and $\gamma^{\prime}$. The level splitting between $|a\rangle$ and $|b\rangle$ (between $|a\rangle$ and $|c\rangle)$ is denoted as $\omega_{a b}\left(\omega_{a c}\right)$. We assume that $|a\rangle$ and $|b\rangle$ are coupled by a weaker 'signal' field with the frequency $\omega$, while $|a\rangle$ and $|c\rangle$ are coupled by a stronger 'control' field with the frequency $\omega^{\prime}$. The detunings of these two transitions are denoted as $\Delta$ and $\Delta^{\prime}$ respectively, which are defined by $\Delta=\omega-\omega_{a b}$ and $\Delta^{\prime}=\omega^{\prime}-\omega_{a c}$. The linear susceptibility of the medium for the weak signal light can be expressed as $[12$, 13, 14

$$
\chi=\chi_{0} \frac{\Gamma \delta\left(\left|\Omega^{\prime}\right|^{2}-4 \Delta \delta+i 2 \delta \Gamma\right)}{\left(\left|\Omega^{\prime}\right|^{2}-4 \Delta \delta\right)^{2}+4 \delta^{2} \Gamma^{2}}
$$

where the constant $\chi_{0}=4 N\left|d_{a b}\right|^{2} /\left(\epsilon_{0} \hbar \Gamma\right), N$ is the density of atomic gas, $d_{a b}$ is the matrix element between the states $|a\rangle$ and $|b\rangle$ of the dipole operator, $\Omega^{\prime}$ is the Rabi frequency of the control light, $\Gamma=\gamma+\gamma^{\prime}$, and $\delta \equiv \Delta-\Delta^{\prime}$ is the two-photon detuning.

In deriving Eq. (1), the one-photon detuning $\Delta$, the twophoton detuning $\delta$, and the Rabi coupling $\Omega^{\prime}$ are assumed to be independent of spatial position. When the external fields exerted on the atomic gas are inhomogeneous, such as in the experiments [6, 9], the above parameters will become spatial dependent, which are denoted as $\Delta(\vec{r}), \delta(\vec{r})$, and $\Omega^{\prime}(\vec{r})$ respectively. Let us assume that the atomic gas cell can be divided into many smaller cells, each smaller cell containing a large number of atoms and the inhomogeneous external field being sufficiently homogenous for each smaller cell. Thus we can apply Eq. (11) to each smaller cell by changing the values of the parameters $\Delta, \delta$, and $\Omega^{\prime}$ for different cells. When the inhomogenous external fields are exerted on the atomic gas, under the above approximation, the linear susceptibility in Eq. (1) will become spatial dependent:

$$
\chi(\vec{r})=\chi_{0} \frac{\Gamma \delta(\vec{r})\left(\left|\Omega^{\prime}(\vec{r})\right|^{2}-4 \Delta(\vec{r}) \delta(\vec{r})+i 2 \delta(\vec{r}) \Gamma\right)}{\left(\left|\Omega^{\prime}(\vec{r})\right|^{2}-4 \Delta(\vec{r}) \delta(\vec{r})\right)^{2}+4 \delta(\vec{r})^{2} \Gamma^{2}} .
$$

To grasp the main physics in Eq. (2), we consider the case where $\delta(\vec{r}), \Delta(\vec{r}) \ll \Gamma \ll\left|\Omega^{\prime}(\vec{r})\right|$. In the first order approximation, the linear susceptibility is simplified to

$$
\chi(\vec{r})=\chi_{0} \frac{\Gamma \delta(\vec{r})}{\left|\Omega^{\prime}(\vec{r})\right|^{2}} .
$$

The vanishing of the imaginary part of the susceptibility in Eq. (3) can be attributed to the steady dark state formed by two lower atomic levels $|b\rangle$ and $|c\rangle$, which completely eliminates the dissipation due to the radiative decay of the excited state $|a\rangle$. Since $\chi(\vec{r}) \ll 1$ near the two-photon resonance, the refraction index is approximated as

$$
n(\vec{r}) \simeq 1+\frac{1}{2} \chi(\vec{r}) .
$$

Once the refraction index $n(\vec{r})$ is known, the trajectory of a light ray propagating in the atomic medium can be obtained by solving the the differential equation [1]

$$
\frac{d}{d s}\left[n(\vec{r}) \frac{d \vec{r}}{d s}\right]=\nabla n(\vec{r}),
$$

where $d s=\sqrt{d x^{2}+d y^{2}+d z^{2}}$. Inserting Eq. (4) into Eq. (5), we get

$$
\frac{d^{2} \vec{r}}{d s^{2}}+\left(\frac{\nabla \chi(\vec{r})}{2} \cdot \frac{d \vec{r}}{d s}\right) \frac{d \vec{r}}{d s}=\frac{\nabla \chi(\vec{r})}{2} .
$$

In the first order approximation, we can apply Eqs. (3) and (6) to determine the light ray trajectory and the corresponding deflection angle.

To demonstrate this procedure, let us consider an example related to the experiments to be studied later. We assume that the signal light injects at the position $\vec{r}_{i}=\left(x_{i}, 0,0\right)$ along $z$ axis. To further simplify the calculation, we approximate the gradient of the linear susceptibility along the light trajectory to be that at the incident point, and the direction of this gradient along $x$ axis, namely,

$$
\frac{\nabla \chi(\vec{r})}{2} \simeq \frac{\nabla \chi\left(\vec{r}_{i}\right)}{2}=\frac{1}{\eta} \vec{e}_{x}
$$

with $\vec{e}_{x}$ being the unit vector along $x$ axis. Then Eq. (6) allows an analytic solution of the light ray path

$$
\begin{aligned}
& x(s)=x(0)+\eta \ln \cosh \frac{s}{\eta}, \\
& y(s)=0, \\
& z(s)=\eta \sinh \frac{s}{\eta} .
\end{aligned}
$$

When the light ray exits the atomic gas cell, we have $z\left(s_{f}\right)=$ $L$, where $L$ is the length along $z$ direction of the atomic gas cell. The length of light path in the atomic medium is then given by $s_{f}=\eta \sinh ^{-1}(L / \eta)$, and we finally arrive at the light deflection angle

$$
\theta \equiv \frac{\dot{x}\left(s_{f}\right)}{\dot{z}\left(s_{f}\right)}=\frac{L / \eta}{1+L^{2} / \eta^{2}} \simeq \frac{L}{\eta},
$$

where the last equality is satisfied only when $L \ll \eta$, which is satisfied throughout this paper. 


\section{LIGHT PROPAGATION IN OPTICALLY CONTROLLED MEDIA}

Here we study the deflection of a weak signal light under a spatially inhomogeneous control light $\Omega^{\prime}(\vec{r})$. At first sight, this model may look similar to that considered in experiment Ref. [9]. However, after carefully examing the experimental parameters, we realized that the experiment was performed using a stronger signal light, for which the linear susceptibility theory is insufficient and the experimental results cannot be explained using our semi-classical theory in the present formulation.

In the absence of an external magnetic field, the two-photon detuning $\delta$ is position independent. We further assume that the driving light has a Gaussian profile

$$
\Omega^{\prime}(\vec{r})=\Omega_{0}^{\prime} \exp \left(-\frac{x^{2}+y^{2}}{\sigma^{2}}\right),
$$

with $\sigma$ characterizing the width of the profile. From Eq. (3), one can easily obtain the linear susceptibility for the signal light to be

$$
\chi(\vec{r})=\chi_{0} \frac{\Gamma \delta}{\left|\Omega_{0}^{\prime}\right|^{2}} \exp \left(2 \frac{x^{2}+y^{2}}{\sigma^{2}}\right) .
$$

An immediate consequence of the above equation is that the light is undeflected if the two photon detuning $\delta$ is set to zero. This result clearly distinguishes our model from the experimentally studied case [9], where a significant light deflection was observed even at resonance. For $\delta \neq 0$ case, we consider the situation where the signal light is sufficiently weak compared to the control light and in the limit $\delta \ll \Omega^{\prime}(\vec{r})$. For the signal lights incident onto the medium at $\vec{r}_{i}=\left(x_{i}, 0,0\right)$ and along the positive $z$-axis within the region $\sqrt{x^{2}+y^{2}} \lesssim \sigma$, we have

$$
\frac{\nabla \chi\left(\vec{r}_{i}\right)}{2}=\chi_{0} \frac{4 \Gamma \delta x_{i}}{\left|\Omega_{0}^{\prime}\right|^{2} \sigma^{2}} \exp \left(\frac{2 x_{i}^{2}}{\sigma^{2}}\right) \vec{e}_{x}=\frac{1}{\eta} \vec{e}_{x} .
$$

Eq. (8) immediately yields the deflection angle

$$
\theta \simeq \chi_{0} \frac{4 \Gamma \delta x_{i} L}{\left|\Omega_{0}^{\prime}\right|^{2} \sigma^{2}} \exp \left(\frac{2 x_{i}^{2}}{\sigma^{2}}\right)
$$

It follows from the above equation that, for $x_{i} \neq 0$, a red detuned signal light $(\delta<0)$ feels an "attractive potential" toward $z$ axis; while a blue detuned light $(\delta>0)$ experiences a "repulsive potential". At $x_{i}=0$, the signal light is undeflected irrespective of its detuning. We also note that the deflection angle increase when $\Omega_{0}^{\prime}$ becomes weaker. These novel light deflection phenomena are schematically illustrated in Fig. 2 .

To get a quantitative idea about the deflection angle, we calculate $\theta$ using the optimal experimental parameters given in Refs. [6, 9]. For example, $\sigma=5 \mathrm{~mm}, L=10 \sigma, \Omega_{0}^{\prime}=5 \Gamma$, and $N=10^{12} / \mathrm{cm}^{3}$. As shown in Fig. 3, we see that the deflection angle becomes larger as the inject position $x_{i}$ increases. Note that this result is valid only when the intensity

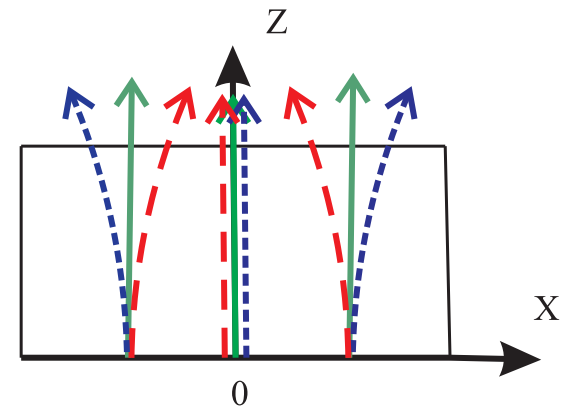

FIG. 2: (Color Online) Schematic illustration about deflection of week signal light in the presence of control light with inhomogeneous profile as in Eq. (9). The three cases with detuning $\delta<0$, $\delta=0$, and $\delta>0$ are denoted by red, green, and blue color respectively. Corresponding to these three cases the light rays with incidents in different positions $x<0, x=0$, and $x>0$ will possesses different deflection ways.

of the local control light $\Omega_{0}^{\prime} \exp \left(-x_{i}^{2} / \sigma^{2}\right) \gg \Gamma$. In addition, the deflection angle increases linearly with the two-photon detuning $\delta$. The deflection angle can reach $0.29 \mathrm{rad}$, which is three orders larger than that in the previous experiment [6]. Thus, these interesting predictions are experimentally observable, and can be explicitly tested by tuning the frequency of the signal light and (or) the incidence position $x$ of the signal light.
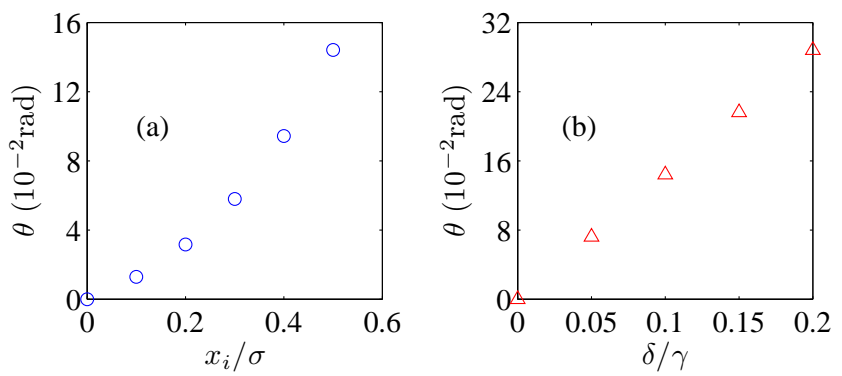

FIG. 3: (Color Online) The deflection angle of the signal light varies with (a) the injection position $x_{i}(\delta=0.1 \Gamma)$, (b) the two-photon detuning $\left(x_{i}=0.5 \sigma\right)$.

As being pointed out previously, the experiment results in Ref. [9] cannot be directly explained using the semiclassical theory based on susceptibility formula Eq. (10). To fully account for the experimental results, a different mechanism based on quantum coherence should be considered. Since both the signal light and the control field are strong, there should be the coherent population trapping for the atoms with the EIT configuration [13], which will result in the nonlinear response for the EIT like effect. It is believed that a refined theoretical approach accounting for the strong signal light, and connecting the quantum interferences of the atomic transitions is required. 


\section{MAGNETICALLY CONTROLLED LIGHT DEFLECTION}

For the next example, we present a semiclassical explanation for the light deflection by a coherent atomic medium subjected to an inhomogeneous magnetic field [6], where the Rabi frequency $\Omega^{\prime}$ are uniform. We consider a linearized inhomogeneous magnetic field $\vec{B}(x)=\left(B_{0}+B_{1} x\right) \vec{e}_{z}$. Thus the two photon detuning

$$
\delta(\vec{r})=\omega-\omega^{\prime}-\mu_{B}\left(B_{0}+B_{1} x\right),
$$

where $\mu_{B}$ is the Bohr magneton, and we used the related spectrum data of ${ }^{87} R b D 1$ line [16]. Thus Eq. (7) gives

$$
\frac{\nabla \chi\left(\vec{r}_{i}\right)}{2}=-\chi_{0} \frac{\Gamma \mu_{B} B_{1}}{2\left|\Omega_{0}^{\prime}\right|^{2}} \vec{e}_{x}=\frac{1}{\eta} \vec{e}_{x} .
$$

In the experiment [6], the light injects onto the atomic gas cell at the point $\vec{r}_{i}=(0,0,0)$ and along $z$ axis. Following Eq. (8), we find the deflection angle to be

$$
\theta=-\chi_{0} \frac{\Gamma \mu_{B} B_{1} L}{2\left|\Omega_{0}^{\prime}\right|^{2}} .
$$

The deflection angle can be reexpressed in terms of group velocity. To this end, we note that

$$
v_{g}=\frac{c}{n+\omega d n / d \omega} \approx \frac{c}{\omega} \frac{2\left|\Omega_{0}^{\prime}\right|^{2}}{\chi_{0} \Gamma},
$$

where we have utilized the fact that

$$
\chi_{0} \frac{\Gamma \omega}{2\left|\Omega_{0}^{\prime}\right|^{2}} \gg 1 .
$$

Inserting (16) into Eq. (15), we get the deflection angle

$$
\theta=-\frac{c \mu_{B} B_{1} L}{v_{g} \omega}
$$

which has exactly the same form as that obtained in Ref. [6] where the control light was treated quantum mechanically. Our calculation, however, indicates that in weak field limit the classical treatment on the control light is capable of capturing the central result on light deflection in an EIT atomic gas.

Note that the concept of group velocity for a light ray does not play any role in our geometric optics method. The unique purpose to derive Eq. (17) is to compare Eq. (15) with the known result in Ref. [6]. Although these two methods give the same result on light deflection angle, the physical picture are quite different. In the picture of dark polariton in Ref. [6, 15], the velocity of dark polariton is the group velocity $c /(n+\omega d n / d \omega)$. In the picture of light ray, however, the signal light with a given frequency propagates with the phase velocity $c / n(\omega)$.

In addition, only transparent medium is considered in geometric optics [17]. This is why we can correctly deal with the signal light deflection in the EIT window, where the atomic gas is transparent to the signal light. However, when we need to further investigate the phenomena on signal light deflection, (for example, considering the signal light frequency region outside of the EIT window, or the energy-conserving dephasing process of the atomic level $|c\rangle$ ), the atomic medium becomes dissipative, and thus the geometric optics method will not be valid any more. A better solution is to directly solve the Maxwell equations in continuous medium, which is beyond the scope of our article.

\section{CONCLUSION}

In summary, we have presented a semi-classical approach to describe the light deflection in the atomic gas cell by applying an inhomogeneous magnetic field or an inhomogeneous pump optical field. Our theory not only explains the experiment without quantization of the probe light, but also predicts some interesting phenomena on quantum coherence enhanced light deflection. The EIT effect not only makes the atomic medium transparent near the two-photon resonance, but also makes the linear susceptibility of the atomic medium spatial dependent. It is this spatial dependent linear susceptibility that deflects the signal light ray. For applications in quantum information processing, the EIT enhanced light deflection can motivate a protocol for quantum sate storage with spatiallydistinguishable channels.

This work is supported by NSFC with grant Nos. 90203018, 10474104, 10674141, and 60433050, and NFRPC with Nos. 2001CB309310, 2005CB724508, 2006CB921206, and 2006AA06Z104. The authors acknowledged helpful discussions with H. Wang, J.X. Zhang, and K.C. Peng.

* Electronic address: zhoud172@ aphy.iphy.ac.cn

$\dagger$ Electronic address: suncp@itp.ac.cn URL: www.itp.ac.cn/ suncp

[1] M. Born and E. Wolf, Principles of Optics, 7th (expanded) edition, Cambridge University Press (1999).

[2] R. Schlesser and A. Weis, Opt. Lett. 17, 1015 (1992).

[3] R. Holzner, et.al., Phys. Rev. Lett. 78, 3451 (1997).

[4] G.T. Purves, G. Jundt, C. S. Adams, and I.G. Hughes, Eur. Phys. J. D 29, 433 (2004).

[5] R.R. Moseley, et. al., Phys. Rev. Lett. 74, 670 (1995).

[6] L. Karpa and M. Weitz, Nature Physics 2, 332 (2006).

[7] S.E. Harris, J.E. Field, and A. Imamoglu, Phys. Rev. Lett. 64, 1107 (1990).

[8] K.-J. Boller, A. Imamoglu, and S. E. Harris, Phys. Rev. Lett. 66, 2593 (1991).

[9] V.A. Sautenkov, H. Li, Y. V. Rostovtsev, and M. O. Scully, quant-ph/0701229

[10] M.D. Lukin, Rev. Mod. Phys. 75, 457(2003).

[11] C.P. Sun, Y. Li, and X.F. Liu, Phys. Rev. Lett. 91, 147903 (2003).

[12] M.O. Scully and M. Fleischauer, Phys. Rev. Lett. 69, 1360 (1992).

[13] M.O. Scully and M. S. Zubairy, Quantum Optics, Cambridge University Press (1997). 
[14] M. Fleischhauer, A. Imamoglu, and J.P. Marangos, Rev. Mod. Phys. 77, 633 (2005).

[15] M. Fleischhauer and M. D. Lukin, Phys. Rev. A 65, 022314 (2002).

[16] See, e.g., D. A. Steck, Rubidium $\left({ }^{87} \mathrm{Rb}\right) \mathrm{D}$ line data, available at http://steck.us/alkalidata

[17] L.D. Landau, E.M. Lifshitz, and L.P. Pitaevskii, Electrodynamics of Continuous Medium, 2nd edition, ButterworthHeinemann Ltd (1984). 